\section{Radioyodo como tratamiento de hipertiroidismo en un paciente en hemodiálisis. Caso clínico}

\author{
CLAUDIA HURTADO ${ }^{1, a}$, MARÍA SOLEDAD BÁEZ ${ }^{2}$, ANABEL BATE², \\ CLAUDIO OPAZO ${ }^{3}$, MAURICIO TRONCOSO $^{3, \mathrm{~b}}$
}

\section{Treatment of hyperthyroidism with radioiodine during hemodialysis. Report of one case}

Although radioiodine $\left({ }^{131}-I\right)$ can be used as treatment of hyperthyroidism for patients in hemodialysis, its use is limited and the experience is mainly related to differentiated thyroid carcinoma. We report a 58 years old female on hemodialysis with recurrent hyperthyroidism after propylthiouracil treatment. She was successfully treated with ${ }^{131}$-I and four months after the intervention her euthyroid state was confirmed. We measured ${ }^{131}$-I activity in blood, dialysate liquid and other waste products, as well as patient radiation exposure rates. We found that ${ }^{131}$-I elimination was prolonged through time with no major dependence on hemodialysis, as opposed to the elimination of ${ }^{131}$-I in patients with thyroid carcinoma. This was probably due to high radiotracer uptake in hyper functioning thyroid tissue. Conversely, radiation content in dialysate wastes or equipment was minimal. Furthermore, the rate of both environmental exposure and exposure of nursing staff in charge of hemodialysis sessions, was minimal and met international security standards. In conclusion, I-131 therapy showed both appropriate effectiveness and safety in this case and may be considered as a suitable treatment alternative to thyroidectomy when antithyroid drugs are unsuccessful.

(Rev Med Chile 2017; 145: 673-677)

Key words: Hemodialysis; Hyperthyroidism; Serum Albumin, Radio-Iodinated.
'Servicio de Medicina Interna,

Hospital Naval Almirante

NEF, Viña del Mar. Escuela de

Medicina, Facultad de Medicina,

Universidad de Valparaíso.

Valparaíso, Chile.

${ }^{2}$ Departamento de

Endocrinología, Servicio de

Medicina Interna, Hospital

Dr. Gustavo Fricke. Viña del Mar,

Chile.

${ }^{3}$ Servicio de Medicina Nuclear,

Hospital Dr. Gustavo Fricke. Viña

del Mar, Chile.

aResidente Medicina Interna.

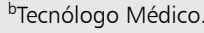

Fuentes de apoyo financiero: No Los autores no declaran conflictos de intereses.

Recibido el 22 de julio de 2016, aceptado el 24 de abril de 2017.

Correspondencia a:

Dra. María Soledad Báez

Álvarez 1532, Viña del Mar.

msoledadbaez@gmail.com

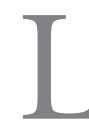

a producción y metabolismo anormal de hormonas tiroideas es relativamente común en pacientes con enfermedad renal crónica terminal (ERCT) y hemodiálisis (HD) $\operatorname{regular}^{1,2}$. El hipotiroidismo, hipotiroidismo subclínico y bocio son entidades más frecuentes en pacientes con ERCT que en la población general, a diferencia del hipertiroidismo que tiene la misma incidencia que en individuos sin ERCT, siendo alrededor del $1,4 \%^{1-4}$. Es esperable entonces, dado el bajo número de casos, que exista escasa experiencia con el uso de radioyodo (131-I) cuando un paciente en hemodiálisis requiere de su administración.
Dado que en pacientes con función renal conservada más del $90 \%$ del 131-I se elimina vía renal $^{1-3}$, los protocolos de administración y selección de la dosis de 131-I en pacientes que están recibiendo hemodiálisis deben ser cuidadosos para minimizar las complicaciones y la exposición del personal a cargo de la terapia dialítica ${ }^{5,6}$.

El caso que se presenta a continuación fue estrechamente monitoreado con el objetivo de medir la tasa de exposición a radiación de la paciente, del personal de salud y la contaminación radiactiva de los equipos de diálisis, para determinar el comportamiento del 131-I en este contexto clínico. 


\section{Caso clínico}

Mujer de 58 años, portadora de enfermedad renal crónica de etiología no precisada desde el año 1995 y en HD trisemanal desde el año 1999. En abril de 2011 se pesquisó TSH frenada de $0,04 \mu \mathrm{IU} / \mathrm{ml}$ en exámenes de rutina, por lo que fue derivada en el año 2012 a evaluación endocrinológica, donde se confirmó hipertiroidismo por manifestaciones clínicas y perfil tiroideo. Se diagnosticó enfermedad de Graves Basedow con cintigrama tiroideo que mostró bocio. No se realizó evaluación de anticuerpos anti receptor de TSH por falta de acceso a este examen. Se trató con propiltiouracilo, único fármaco antitiroideo disponible en nuestro establecimiento al momento del diagnóstico, este tratamiento se mantuvo durante 30 meses, logrando el eutiroidismo por lo que se retiró en forma progresiva. A los 5 meses de suspendido el medicamento, se observa nuevamente TSH suprimida, pero con T3 y T4 normales, por lo que se decidió administrar tratamiento con 131-I.

La paciente recibió 17 mCi de 131-I en cápsula (como yoduro de sodio), inmediatamente después de una sesión completa de HD y permaneció hospitalizada en aislamiento radiactivo durante los 7 días siguientes. No presentó complicaciones secundarias al tratamiento. Cabe señalar que esta paciente presentaba una diuresis residual, cercana a los 400 cc/día.

La primera HD posterior a la administración de 131-I se realizó a las 90 h, con un equipo Fre- senius 4008s y filtro de polietersulfona (PES) 210, con una velocidad de flujo sanguíneo de $300 \mathrm{ml} /$ min (QB), flujo de dializado de $500 \mathrm{ml} / \mathrm{min}$ (QD), durante $3 \mathrm{~h}(\mathrm{QT})$, lográndose un ultrafiltrado (UF) neto de $1.900 \mathrm{ml}$. La segunda HD fue a las $46 \mathrm{~h}$ de la primera ( $6^{\circ}$ día), con el mismo equipo, con un filtro PES 170, utilizando los mismos parámetros de QB, QD y QT, obteniéndose un UF neto de $2.000 \mathrm{ml}$.

Se realizaron mediciones de exposición a radiación con medidor Geiger Muller Fluke Biomedical modelo 489-110D en contacto directo con el tiroides, a 1 y 1,5 metros de la paciente en los días expuestos en la Tabla 1, donde se muestran las tasas obtenidas. Durante las sesiones de HD se midió la tasa de exposición a la que estuvo expuesta la enfermera a cargo y el equipo de diálisis; además se tomaron alícuotas de $1 \mathrm{ml}$ de sangre y líquido dializado para medir la actividad de 131-I en Mega Becquerel (MBq), con activímetro marca Alfa Nuclear modelo ACT-15P, así se estimó la eliminación por sesión de HD.

Las tasas de exposición a radiación luego de la primera y segunda $\mathrm{HD}$, fueron $0 \mu \mathrm{Sv} / \mathrm{h}$ y $0,3 \mu \mathrm{Sv} / \mathrm{h}$ para el equipo, y de $3 \mu \mathrm{Sv} / \mathrm{h}$ y $0,36 \mu \mathrm{Sv} / \mathrm{h}$, para el filtro de diálisis, respectivamente.

La medición de exposición de la enfermera a cargo de las sesiones de HD se realizó con un dosímetro de lectura directa RAD-51/60/62, lo que resultó en $1,0 \mathrm{mR}(10 \mu \mathrm{Sv})$ durante la primera sesión de $\mathrm{HD}(4 \mathrm{~h})$ y $0,6 \mathrm{mR}$ la segunda (sólo expuesta $1 \mathrm{~h}$ ). Cabe destacar que la enfermera utilizó delantal plomado y se sitúo tras un biombo

Tabla 1. Tasa de exposición a radiación en diferentes distancias desde la administración de $17 \mathrm{mCi}$ de 131-I al día 10 en paciente hipertiroidea en hemodiálisis

\begin{tabular}{|c|c|c|c|}
\hline & $\begin{array}{c}\text { Tasa exposición en } \\
\text { contacto con tiroides } \\
\text { (mSv/h) }\end{array}$ & $\begin{array}{c}\text { Tasa exposición a } 1 \\
\text { metro de la paciente } \\
\text { (mSv/h) }\end{array}$ & $\begin{array}{l}\text { Tasa exposición a 1,5 } \\
\text { metros de la paciente } \\
\text { (mSv/h) }\end{array}$ \\
\hline Inmediatamente post 131-I* & 0,433 & 0,043 & 0,015 \\
\hline 48 h post administración* & 1,65 & 0,071 & 0,022 \\
\hline Previo a la $1^{\mathrm{a}} \mathrm{HD} \mathrm{D}^{\S}$ & 3,9 & 0,054 & 0,021 \\
\hline Posterior a la $1^{\mathrm{a}} \mathrm{HD} \S$ & 3,8 & 0,042 & 0,016 \\
\hline Previo a la $2^{\mathrm{a}} \mathrm{HD}^{\S}$ & 3,3 & 0,032 & 0,013 \\
\hline Posterior a la $2^{\mathrm{a}} \mathrm{HD} D^{\S}$ & 3,7 & 0,028 & 0,012 \\
\hline 10 días post $131-\left.\right|^{\S}$ & 2,8 & 0,013 & 0,008 \\
\hline
\end{tabular}

131-I: radioyodo 131; mCi: miliCurie; mSv/h: miliSievert por hora. *Dato calculado a partir de medición en CPM (cuentas por minuto). ${ }^{\circledR}$ Determinaciones realizadas con medidor GeigerMullerFlukeBiomedical modelo 489-110D. 
Tabla 2. Actividad de 131-I medida en sangre, en dializado y en agua de lavado, de una paciente hipertiroidea en hemodiálisis posterior a una dosis de $17 \mathrm{mCi}$ de 131-I

\begin{tabular}{|c|c|c|c|}
\hline & \multicolumn{2}{|c|}{ Actividad de 131-I } \\
\hline & & $\mathbf{m C i}$ & $\mathbf{M B q}$ \\
\hline \multirow[t]{3}{*}{ Sangre de $1^{\mathrm{a}} \mathrm{HD}$} & Pre & 0,3 & 0,0111 \\
\hline & $1 \mathrm{~h}$ & 0,2 & 0,0074 \\
\hline & Post & 0,4 & 0,0148 \\
\hline Dializado de $1^{\text {a }} \mathrm{HD}$ & & 0,2 & 0,0074 \\
\hline Agua de lavado de $1^{\mathrm{a}} \mathrm{HD}$ & & 0 & 0 \\
\hline \multirow[t]{3}{*}{ Sangre de $2^{\mathrm{a}} \mathrm{HD}$} & Pre & 0 & 0 \\
\hline & $2^{a}$ hora & 0 & 0 \\
\hline & Post & 0 & 0 \\
\hline Dializado de $2^{\circ} \mathrm{HD}$ & & 0 & 0 \\
\hline Agua de lavado de $2^{\circ} \mathrm{HD}$ & & 0 & 0 \\
\hline
\end{tabular}

131-I: Radioyodo 131; mCi: miliCurie; MBq: MegaBecquerel HD: Hemodiálisis. Instrumento de medición: Activímetro Alfa Nuclear modelo ACT-15P.

blindado cuando no estaba en contacto directo con la paciente.

Respecto a la actividad de 131-I medida en sangre y líquido de dializado antes, durante y después de las sesiones de HD se resumen en la Tabla 2.

$\mathrm{Al}$ mes después de la terapia los parámetros de función tiroidea aún mostraban hipertiroidismo con TSH de $0,004 \mu \mathrm{IU} / \mathrm{ml}$ (VN: 0,27-4,2) y T4 libre de 2,07 ng/dl (VN: 0,9-1,7). Luego de 4 meses se observó normalización de la TSH $(2,31 \mu \mathrm{IU} / \mathrm{ml})$ y descenso de la T4 libre $(0,730 \mathrm{ng} / \mathrm{ml})$, lo que confirmó la efectividad del tratamiento, posterior a esto la paciente continuó controles por la alta probabilidad de requerir terapia de sustitución con levotiroxina.

\section{Discusión}

El uso de 131-I para el tratamiento del hipertiroidismo en pacientes en hemodiálisis es poco frecuente y no existen protocolos estandarizados para su realización. La mayor parte de la experiencia mundial reportada es referente a pacientes con carcinoma diferenciado de tiroides (CDT) tratado con131-I durante HD. Sin embargo, los datos no son extrapolables, puesto que las dosis utilizadas y la farmacodinamia del 131-I en pacientes tiroidectomizados son distintas a la de pacientes hipertiroideos, lo que se explica por la captación del radiofármaco en la glándula tiroidea ${ }^{5-8}$.

Normalmente la eliminación del 131-I depende fundamentalmente del aclaramiento renal, con tasas de eliminación de radioactividad del $80 \%$ a las $48 \mathrm{~h}$ de la administración ${ }^{2,6,7}$, siendo la vida media efectiva del 131-I de 14 a 24 h para CDT operado o 4 a 5 días en hipertiroidismo ${ }^{1,7,8}$.

En pacientes con ERCT en HD el 131-I presenta un comportamiento distinto, tanto en CDT como en hipertiroidismo. Estudios han mostrado que la actividad máxima del 131-I se produce al segundo día post administración ${ }^{3,6-8}$. La tasa de eliminación después de cada sesión de HD es menor en hipertiroidismo que en CDT (2,7-5,5\% versus $50-70 \%$, respectivamente) $)^{3,11,12}$, determinando tiempos de vida media efectiva en pacientes hipertiroideos de 6,5-7,1 días $3,11,12$.

En este caso se utilizó una dosis estándar de 131-I para hipertiroidismo, teniendo en consideración que, a diferencia de lo que ocurre en pacientes tiroidectomizados por CDT, la dosis requerida para tratamiento del hipertiroidismo es significativamente menor, además de que esta dosis sería captada en su gran mayoría por el tejido tiroideo sin exponer a exceso de radiación a otros órganos; por otra parte, se estimó una dosis suficiente para tratar en forma definitiva el hipertiroidismo y evitar la necesidad de una segunda dosis de 131-I.

Nuestra experiencia se asemeja a lo descrito, ya que la mayor tasa de exposición se registró después de las $24 \mathrm{~h}$ de la administración del 131-I, siendo máxima en las mediciones directas sobre tiroides, lo que demostró concentración del radiofármaco en el tejido tiroideo. La tasa de exposición a distancia evidenció una curva descendente con una pendiente poco pronunciada (Figura 1), lo que refleja una tasa de eliminación menor, con registros de exposición a radiación de $0,013 \mathrm{mSv} / \mathrm{Hr}$ a 1 metro y $69 \%$ de disminución de radioactividad al décimo día de la administración, comparable con las series de Homer et al y Simpson et al ${ }^{11,12}$.

La baja actividad de 131-I medida en sangre y líquido de dializado antes, durante y después de las sesiones de HD refleja la baja tasa de exposición de los tejidos extratiroideos y la baja tasa de eliminación en líquido de dializado, respectivamente, esto también se explica por la mayor captación del 131I por el tejido tiroideo, lo que determina menor 


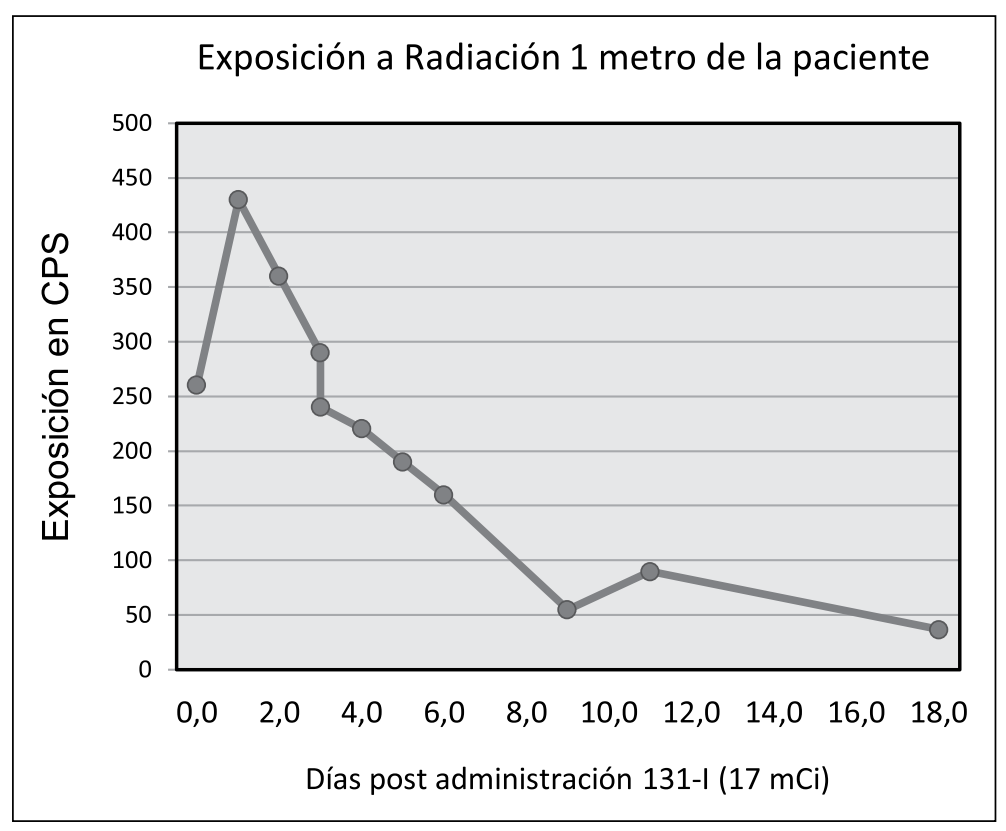

Figura 1. Tasa de exposición a radiación a 1 metro de una paciente hipertiroidea en hemodiálisis desde la administración de 17 mCi de 131-I hasta los 18 días. CPS: cuentas por segundo; 131-I: Radioyodo 131; mCi: miliCurie. Instrumento de medición: Dosímetro de lectura directa RAD-51/60/62. concentración del radiofármaco en circulación y menor eliminación de éste durante la HD, lo que difiere de lo observdo en CDT.

Durante la HD tanto el filtro como el equipo de diálisis tienen contacto directo con el paciente y su contenido vascular, sin embargo, las tasas de exposición encontradas en estos dispositivos son mínimas, lo que concuerda con lo publicado en otras series $^{5,6,9}$. Así mismo, pareciera que con las medidas de seguridad estándar, la exposición del personal a cargo del paciente es mínima, asemejándose a la radiación recibida por una radiografía de rodillas o tórax ${ }^{14,15}$, lo que está dentro de lo permitido en las normas nacionales e internacionales para personas que no trabajan habitualmente con radiación y que es de $1 \mathrm{mSv} / \mathrm{añon}^{16,17}$.

En conclusión, el administrar 17 mCi de 131I como tratamiento para el hipertiroidismo en este caso resultó efectivo y seguro, lográndose el control de la enfermedad, lo que nos permite considerar este tratamiento como una excelente alternativa a la tiroidectomía para los pacientes en hemodiálisis.

Agradecimientos: Se extienden nuestros agradecimientos al Departamento de Nefrología del
Hospital Dr. Gustavo Fricke de Viña del Mar, en especial a la Dra. Beatriz Tapia y a la E. U. Srta. Roxana Cabero, por su valiosa colaboración.

\section{Referencias}

1. Mohamedali M, Reddy Maddika S, Vyas A, Iyer V, Cheriyath P. Thyroid Disorders and Chronic Kidney Disease. Int J Nephrol 2014; 2014 (4): 1-6.

2. Iglesias P, Díez JJ. Thyroid dysfunction and kidney disease. Eur J Endocrinol 2009; 160 (4): 503-15.

3. Boubaker K, Hayet K, Ben Maiz H, Kheder A. Characteristics of Graves' Disease in Haemodialysis Patients. J Endocrinol Metab 2012; 2 (6): 212-5.

4. Encuesta Nacional de Salud ENS Chile 2009-2010. Ministerio de Salud. Gobierno de Chile. Disponible en Ministerio de Salud. Encuesta Nacional de Salud ENS Chile 2009-2010. Disponible en: http://web.minsal.cl/ portal/url/item/bcb03d7bc28b64dfe040010165012d23. pdf [consultado el 23 de enero de 2016].

5. Modarresifar H, Almodovar S, Bass WB, Ojha B. Radiation safety protocol for high dose 131I therapy of thyroid carcinoma in patients on hemodialysis for chronic renal failure. Health Phys 2007; 92 (2 Suppl): S45-9. 
6. Saracyn M, Bilski M, Kamiński G, Niemczyk S. Can radioiodine be administered effectively and safely to a patient with severe chronic kidney disease? Clin Endocrinol 2014; 81 (2): 169-74.

7. Holst J, Burman K, Atkins F, Umans J, Jonklaas J. Radioiodine Therapy for Thyroid Cancer and Hyperthyroidism in Patients with End-Stage Renal Disease on Hemodialysis. Thyroid 2005; 15 (12): 1321-31.

8. Mulazimoglu M, Edis N, Tamam MO, Uyanik E, Ozpacaci $\mathrm{T}$. The evaluation of the external dose measurement of the patients treated with radioiodine therapy. Radiat Prot Dosimetry 2010; 141 (3): 233-8.

9. Gallegos-Villalobos A, García-López F, Escalada C, Ortiz JJ, Cardona J, Medina A, et al. Uso de yodo radiactivo I-131 y monitorización de radiactividad en pacientes con enfermedad renal crónica en hemodiálisis. Nefrologia 2014; 34 (3): 317-22.

10. Sinsakul M, Ali A. Radioactive 131I Use in End-Stage Renal Disease: Nightmare or Nuisance? Semin Dial 2004; 17 (1): 53-6.

11. Simpson JB, Godwin GA. Radiation safety implications of 131I treatment in a patient with Grave's disease undergoing home hemodialysis. Health Phys 2006; 91
(6): 570-3.

12. Homer L, Smith AH. Radiation protection issues of treating hyperthyroidism with $131 \mathrm{I}$ in patients on haemodialysis. Nucl Med Commun 2002; 23 (3): 261-4.

13. Alevizaki C, Molfetas M, Samartzis A, Vlassopoulou B, Vassilopoulos C, Rondogianni PH, et al. Iodine 131 treatment for differentiated thyroid carcinoma in patients with end stage renal failure: dosimetric, radiation safety, and practical considerations. Hormones (Athens) 2006; 5 (4): 276-87.

14. Murcutt G, Edwards J, Boakye J, Davenport A. Hemodialysys of chronic kidney failure patients requiring ablative radioiodine therapy. Kidney Int 2008; 73 (11): 1316-9.

15. Andisco D, Blanco S, Buzzi AE. Dosimetría en radiología. Rev Argent Radiol 2014; 78 (2): 114-7.

16. Vigilancia Radiológica Ocupacional. Instituto de Salud Pública, Ministerio de Salud. Gobierno de Chile. Disponible en http://www.ispch.cl/vigilancia-radiologica-ocupacional [consultado el 1 de marzo de 2016].

17. Report No. 116 - Limitation of Exposure to Ionizing Radiation. National Council on Radiation Protection and Measurement. NCRP publication, 1993. 